\title{
THE COMPLETE EXISTENTIAL THEORY OF HURWITZ'S POSTULATES FOR ABELIAN GROUPS AND FIELDS*
}

BY B. A. BERNSTEIN

1. Introduction. Hurwitz has proposed sets of postulates for abelian groups and fields. $\dagger$ If $F^{\prime}, F^{\prime \prime}, F_{n}$ denote his sets for denumerable, continuous, and finite fields respectively, $G^{\prime}, G^{\prime \prime}, G_{n}$ the corresponding sets for abelian groups, then I have proved in another paper $\ddagger$ the following theorem.

Theorem A. Postulate-sets $F^{\prime}, F^{\prime \prime}, G^{\prime}, G^{\prime \prime}, G_{n}(n>1)$ are each completely independent; postulate-set $F_{n}$ is completely independent $\$$ when, and only when, $n$ exceeds 2 and is a power of a prime.

The object of this note is to investigate postulate-set $F_{n}$ further, and to prove the following theorem, which, together with Theorem $A$ establishes the complete existential theory $\$$ of each of Hurwitz's six postulate-sets for abelian groups and fields.

Theorem $B$. For postulate-set $F_{n}$, when $n$ exceeds 2 and is not a power of a prime, there exists no system having the character $(++++++)$, but there exist systems having all the other characters; when $n=2$ there exist no systems having the characters $(-+++-+)$ and $(-+-+-+)$, but there exist systems having all the other characters.

2. Hurwitz's Postulates $F_{n}$ for Finite Fields. For finite fields Hurwitz's postulates are as follows $(K, \oplus, \odot$ being undefined): $\left(A_{1}\right)$ If $a, b, c, a \oplus b, c \oplus b$, and $a \oplus(c \oplus b)$ belong to $K$, then $(a \oplus b) \oplus c=a \oplus(c \oplus b)$.

* Presented to the Society April 8, 1922.

$\dagger$ W. A. Hurwitz, Postulate-sets for abelian groups and fields, Annals of Mathematics, (2), vol. 15 (1913), p. 93.

$\ddagger$ On complete independence of Hurwitz's postulates for abelian groups and fields, Annals of Mathematics, (2), vol. 24 (1922).

$\S$ See E. H. Moore, Introduction to a form of general analysis, New Haven Mathematical Colloquium, Yale University Press, p. 82. 
( $\left.A_{2}\right)$ If $a$ and $b$ belong to $K$, then there is an element $x$ of $K$ such that $a \oplus x=b$.

$\left(M_{1}\right)$ If $a, b, c, a \odot b, c \odot b$, and $a \odot(c \odot b)$ belong to $K$, then $(a \odot b) \odot c=a \odot(c \odot b)$.

$\left(M_{2}\right)$ If $a$ and $b$ belong to $K$, and $a \oplus a \neq a$, there is an element $x$ of $K$ such that $a \odot x=b$.

(D) If $a, b, c, a \odot b, a \odot c, b \oplus c,(a \odot b) \oplus(a \odot c)$ belong to $K$, then $a \odot(b \oplus c)=(a \odot b) \oplus(a \odot c)$.

$\left(N_{n}\right) K$ contains $n>1$ elements.*

3. Proof of Theorem B. The proof of Theorem $B$ is obtained with the help of the table below. $\dagger$

(1) Systems 1-32 of the table have the characters ( $\pm \pm \pm \pm \pm 一)$ for $F_{n}$.

(2) Let $1^{\prime}-32^{\prime}$ be the systems obtained from 1-32 by substituting (a) the class of least positive residues modulo $n$ for the class of integers and $(b)$ the least positive residue modulo $n$ of $a+b, a b, a-b$ for $a+b, a b$, and $a-b$ respectively, except that $a^{2}+(b-1)^{2}$ in 16 is left unchanged. Then when $n>2$, systems $2^{\prime}-32^{\prime}$ will have the characters $( \pm \pm \pm \pm \pm+)$ except $(++++++)$; when $n=2$, systems $1^{\prime}-9^{\prime}, 11^{\prime}-20^{\prime}, 22^{\prime}-32^{\prime}$ will be systems having all the characters $( \pm \pm \pm \pm \pm+)$ except $(-+++-+)$ and $(-+-+-+)$.

(3) When $n>2$ and not a power of a prime there exists no field.

(4) When $n=2$ there exists no system having the character $(-+++-+)$. For, since postulates $\left(A_{1}\right)$ and $(D)$ have to be contradicted, and $\left(A_{2}\right)$ satisfied, $a \oplus b$ must be defined by the table

\begin{tabular}{l|ll}
$\oplus$ & 0 & 1 \\
\hline 0 & 1 & 0 \\
1 & 1 & 0
\end{tabular}

Further, since postulates $\left(M_{1}\right)$ and $\left(M_{2}\right)$ have to be satisfied,

* Postulate-sets $F^{\prime}$ and $F^{\prime \prime}$ are obtained from set $F_{n}$ by substituting for $\left(N_{n}\right)$ respectively: $\left(N^{\prime}\right) K$ is countably infinite, $\left(N^{\prime \prime}\right) K$ has the cardinal number of the continuum. Sets $G_{n}, G^{\prime}, G^{\prime \prime}$ are obtained from $F_{n}, F^{\prime}, F^{\prime \prime}$ respectively by omitting $\left(M_{1}\right),\left(M_{2}\right)$, and $(D)$.

$\dagger$ This table may also be used conveniently in the proof of Theorem $A$. 
$a \odot b$ must be defined by one or the other of the tables:

\begin{tabular}{c|cc}
$\odot$ & 0 & 1 \\
\hline 0 & 1 & 0 \\
1 & 0 & 1
\end{tabular}

\begin{tabular}{c|cc}
$\odot$ & 0 & 1 \\
\hline 0 & 0 & 1 \\
1 & 1 & 0
\end{tabular}

That is, we must have either

$$
a \oplus b=b+1(\bmod 2), \quad a \odot b=a+b+1(\bmod 2)
$$

or else

$$
a \oplus b=b+1(\bmod 2), \quad a \odot b=a+b(\bmod 2) .
$$

But for either case postulate $(D)$ would be satisfied.

(5) That when $n=2$ there is no system having the character $(-+-+-+)$ I have shown in the paper cited above.

This completes the proof of our theorem.

\begin{tabular}{|c|c|c|c|c|}
\hline No. & Character & $K$ & $a \oplus b$ & $a \odot b$ \\
\hline $\begin{array}{l}17 \\
18 \\
19 \\
20 \\
21 \\
22 \\
23 \\
24 \\
25 \\
26 \\
27 \\
28 \\
29 \\
30 \\
31 \\
32\end{array}$ & 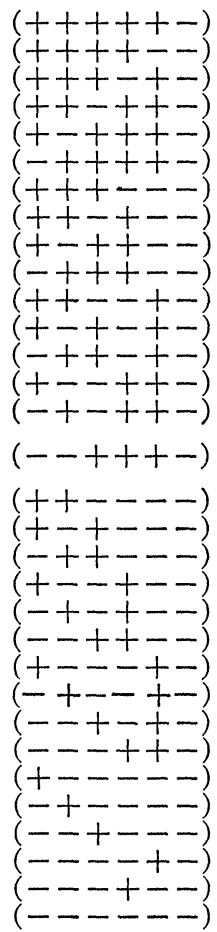 & $\begin{array}{c}\text { Integers* } \\
\text { "6 } \\
6 \\
\text { "6 } \\
6 \\
\text { "6 } \\
6 \\
6 \\
6 \\
6 \\
6 \\
6 \\
6 \\
6 \\
4 \\
6 \\
6 \\
6 \\
6 \\
6 \\
6 \\
6 \\
6 \\
6 \\
6 \\
6 \\
6 \\
6 \\
6\end{array}$ & $\begin{array}{c}a+b \\
a+b \\
a+b \\
a+b \\
a \\
b \\
a+b \\
a+b \\
0 \\
a-b \\
a+b+1 \\
0 \\
b+1 \\
0 \\
b \\
+0 \\
a+(b-1)^{2} \\
a+b \\
0 \\
b+1 \\
0 \\
a-b \\
a b+a \\
1 \\
b+1 \\
a b+a \\
a+1 \\
0 \\
b+1 \\
a+1 \\
b+0 / a \\
a b+a \\
a+1\end{array}$ & $\begin{array}{l}a b \\
a+b \\
0 \\
b \\
a+b \\
a+b \\
1 \\
b+1 \\
a+b \\
a+b \\
b+0 / a \\
0 \\
a / 0 \\
b \\
b \\
a \\
a+1 \\
1 \\
1 \\
b+1 \\
a-b \\
a+b \\
b+0 / a \\
b+0 / a \\
0 \\
b \\
a+1 \\
a+1 \\
a \\
b+0 / a \\
b+1 \\
a+1\end{array}$ \\
\hline
\end{tabular}

Sxstems Having the Characters ( $\pm \pm \pm \pm \pm 一$ ) for $F_{n}$

* Positive, negative, and 0.

The University of California 